\title{
Variation in the use of observation status evaluation in Massachusetts acute care hospitals, 2003-2006
}

\author{
Jeremiah D. Schuur • Arjun K. Venkatesh • \\ J. Stephen Bohan
}

Received: 10 March 2010 / Accepted: 19 April 2010 /Published online: 20 August 2010

(C) Springer-Verlag London Ltd 2010

\begin{abstract}
Background Observation evaluation is an alternate pathway to inpatient admission following Emergency Department (ED) assessment.

Aims We aimed to describe the variation in observation use and charges between acute care hospitals in Massachusetts from 2003 to 2006.

Methods Retrospective pilot analysis of hospital administrative data. Patients discharged from a Massachusetts hospital between 2003 and 2006 after an observation visit or inpatient hospitalization for six emergency medical conditions, grouped by the Clinical Classification System (CCS), were included. Patients discharged with a primary obstetric condition were excluded. The primary outcome measure, "Observation Proportion" (pOBS), was the use of observation evaluation relative to inpatient evaluation (pOBS $=\mathrm{n}$ Observation/(n Observation $+\mathrm{n}$ Inpatient). We calculated pOBS, descriptive statistics of use and charges by the hospital for each condition.

Results From 2003 to 2006 the number of observation visits in Massachusetts increased 3.9\% [95\% confidence
\end{abstract}

J. D. Schuur $(\varangle) \cdot$ J. S. Bohan

Department of Emergency Medicine,

Brigham and Women's Hospital,

75 Frances St. Neville House,

Boston, MA 02115, USA

e-mail: jschuur@partners.org

J. D. Schuur · J. S. Bohan

Division of Emergency Medicine, Harvard Medical School, Boston, MA, USA

A. K. Venkatesh

Harvard Affiliated Emergency Medicine Residency-Brigham and Women's Hospital-Massachusetts General Hospital, Boston, MA, USA interval (CI) $3.8 \%$ to $4.0 \%$ ] from 128,825 to 133,859 , while inpatient hospitalization increased $1.29 \%$ (95\% CI $1.26 \%$ to $1.31 \%$ ) from 832,415 to 843,617 . Nonspecific chest pain (CCS 102) was the most frequently observed condition with 85,843 ( $16.3 \%$ of total) observation evaluations. Observation visits for nonspecific chest pain increased $43.5 \%$ from 2003 to 2006 . Relative observation utilization (pOBS) for nonspecific chest pain ranged from $25 \%$ to $95 \%$ across hospitals. Wide variation in hospital use of observation and charges was seen for all six emergency medical conditions.

Conclusions There was wide variation in use of observation across six common emergency conditions in Massachusetts in this pilot analysis. This variation may have a substantial impact on hospital resource utilization. Further investigation into the patient, provider and hospital-level characteristics that explain the variation in observation use could help improve hospital efficiency.

Keywords Emergency service $\cdot$ Hospital $\cdot$ Small-area analysis $\cdot$ Hospital charges $\cdot$ Chest pain

\section{Introduction}

Observation care is an alternative to inpatient admission designed for evaluation and management of patients during a short stay, usually defined as between 6 and 24 hours [1]. Interest in observation medicine has increased over the past decade, and the variety of clinical conditions considered suitable for observation has expanded from initial pathways for asthma and chest pain to a wider set of conditions such as transient ischemic attack and syncope [1,2]. Despite growing clinical research on observation protocols, there are no published data describing the utilization and 
variation of observation care for common emergency medical conditions.

Inpatient hospitalization provides intensive clinical care but is the most expensive, capacity-limited and potentially dangerous location for health care delivery [3, 4]. Inpatient hospitalization rates vary across the US in patterns that cannot be explained solely by patients' medical history or clinical condition, but do correlate with health system factors, such as the supply of inpatient beds [5]. Given that half of all inpatients are admitted through hospital EDs, emergency physicians play a major role in the utilization of inpatient hospitalization. Chest pain alone accounted for nearly 7 million ED visits and 800,000 hospitalizations in 2006, making it the second most frequent ED chief complaint and hospital discharge diagnosis [6]. From 1997 to 2005 the costs of inpatient care for nonspecific chest pain grew $181 \%$ to over $\$ 10$ billion annually [7]. Appropriate use of observation care can reduce the use of inpatient hospitalization as validated observation protocols have demonstrated similar or better clinical outcomes at lower costs for a number of common emergency medical conditions [1,2]. Despite this, there is a dearth of research on the use of observation for emergency medical conditions across systems of care.

We aimed to describe the variation in use of observation evaluation and observation charges between acute care hospitals in Massachusetts from 2003 to 2006. Our primary objective was to describe the frequency of observation visits relative to inpatient hospitalization for common emergency medical conditions. Additionally, we aimed to describe the potential reduction in hospital charges associated with use of observation services in place of inpatient hospitalization for nonspecific chest pain. We hypothesized that there is clinically important variation in the use of observation evaluation for common emergency medical conditions across hospitals.

\section{Materials and methods}

Study design, setting and selection of participants

This was a retrospective analysis of hospital administrative data. All Massachusetts acute care hospitals submit standardized utilization data for each inpatient hospitalization, ED and observation visit to the Department of Health and Human Services Division of Health Care Finance and Policy (DHCFP). Hospital-level summary utilization data files are available to the public without patient identifiers for both inpatient and observation evaluations. Observation visits are defined as discharges from observation status with a charge for observation. Inpatient hospitalizations are defined as hospital discharges with a recorded charge for inpatient stay. The two definitions are mutually exclusive; patients admitted to inpatient from observation status are defined as inpatients.

Inclusion criteria

Patients with an observation visit or an inpatient hospitalization in a Massachusetts acute care hospital from 2003 to 2006 were included. Patients evaluated and discharged from the ED without a subsequent observation visit or inpatient hospitalization were not included. We included the five most frequently observed emergency medical conditions, grouped by Clinical Classification System (CCS; Table 1) [8]. Additionally, we included congestive heart failure (CCS 108) in the analysis because prior to 2007, it was one of three clinical conditions, asthma (CCS 128) and nonspecific chest pain (CCS 102) being the others, designated for payment by the Centers for Medicare and Medicaid Services, and therefore was hypothesized to have high observation utilization.

\section{Exclusion criteria}

Of the eight most frequently observed CCS conditions, we excluded the three conditions unlikely to represent common emergency medical observation pathways. Patients with primary obstetric diagnoses were excluded (other complications of pregnancy, CCS 181; early or threatened labor, CCS 184), as most such patients are seen in labor and delivery units rather than EDs. Patients with cardiac dysrhythmias (CCS 106) were excluded as many such patients may undergo observation outside of the $\mathrm{ED}$ for elective procedures. Hospitals with fewer than 20 observation visits for a CCS condition in a calendar year had that year's data excluded from hospital-level analysis as samples less than 20 do not provide stable estimates [9].

Data collection and processing

We obtained summary utilization files for all Massachusetts acute care hospitals that reported to the DHCFP from 2003 to 2006. Between 2003 and 2006, the total number of hospitals reporting inpatient data decreased from 77 to 74 , while the number reporting observation visits declined from 74 to 70 , both as a result of hospital merger or closure. The DHCFP checks each hospital's data for integrity, cleans the data and applies validated algorithms to group diagnoses into clinical conditions, including the CCS and diagnosisrelated group (DRG). The CCS is a validated algorithm that groups primary International Classification of Diseases version 9, Clinical Modification (ICD-9) discharge diagnoses into 285 clinical categories [8]. DRG 143, including inpatient discharge ICD-9 codes associated with chest pain 
Table 1 Utilization of observation evaluation for common emergency conditions, Massachusetts 2003-2006

\begin{tabular}{|c|c|c|c|c|c|c|}
\hline Clinical condition $(\mathrm{CCS})(6)$ & $\begin{array}{l}\text { Hospitals } \\
\mathrm{n}\end{array}$ & $\begin{array}{l}\text { Patients } \\
\text { observed } \\
\mathrm{n} \mathrm{Median} \\
(\min , \max )\end{array}$ & $\begin{array}{l}\text { Inpatient } \\
\text { discharges } \\
\mathrm{n} \text { Median } \\
(\min , \max )\end{array}$ & $\begin{array}{l}\text { pOBS } \\
\% \text { Median } \\
(\min , \max )\end{array}$ & $\begin{array}{l}\text { Trend across } \\
\text { years } \\
P \text { q }\end{array}$ & $\begin{array}{l}\text { Observation } \\
\text { charges }^{\mathrm{a}} \\
\text { Median, } \\
\text { \$ (min, max) }\end{array}$ \\
\hline $\begin{array}{l}\text { Nonspecific chest } \\
\text { pain }(102)\end{array}$ & 74 & $912(50,4,686)$ & $680(15,3,474)$ & $59.0 \%(25.1,94.9)$ & $<0.0001$ & $\$ 5,438(3,104,11,633)$ \\
\hline $\begin{array}{l}\text { Fluid and electrolyte } \\
\text { disorders (55) }\end{array}$ & 59 & $112(20,760)$ & $416(21,2,077)$ & $21.8 \%(8.3,69.5)$ & $<0.0001$ & $\$ 3,910(2,116,8,464)$ \\
\hline Syncope (245) & 63 & $189(25,1,112)$ & $348(22,1,549)$ & $37.7 \%(14.2,81.9)$ & $<0.0001$ & $\$ 5,658(2,468,11,745)$ \\
\hline Abdominal pain (251) & 58 & $142(20,2,337)$ & $208(3,737)$ & $47.5 \%(17.7,88.5)$ & 0.56 & $\$ 5,361(2,840,11,530)$ \\
\hline Asthma (128) & 39 & $138(21,964)$ & $425(62,2,820)$ & $22.4 \%(7.1,51.4)$ & $<0.0001$ & $\$ 3,784(2,174,6,401)$ \\
\hline $\begin{array}{l}\text { Congestive heart failure, } \\
\text { non-hypertensive (108) }\end{array}$ & 19 & $62(20,188)$ & $503(39,1,919)$ & $8.4 \%(4.6,35)$ & 0.26 & $\$ 5,370(2,867,27,395)$ \\
\hline
\end{tabular}

q $P$ values are based on the Mantel-Haensel test for trend

${ }^{\text {a }}$ Observation charges from 2005

without clear cardiac, toxic, or operative etiology, was used to compare charges between observation and inpatient [10]. The DHCFP links observation visit codes to inpatient hospitalization codes to ensure appropriate comparison of frequency data.

\section{Outcome measures}

Our primary outcome was the "Observation Proportion" (pOBS), defined as the percent of patients who had an observation visit among all patients with an observation visit or inpatient hospitalization for a CCS condition:

$p O B S=n$ Observation $/(n$ Observation $+n$ Inpatient $)$

Secondary outcomes include total observation and inpatient charges for each CCS condition at the hospital level.

Data analysis

We summed the number of observation visits for each of the 20 most frequent CCS conditions and ranked conditions by frequency. We then calculated pOBS for each of the six included CCS conditions (defined above) for each Massachusetts hospital. To illustrate variation, we report the median hospital's observation and inpatient visit census, pOBS and the range across hospitals.

We compared the number of observation and inpatient visits by year using the Mantzel-Haensel test for trend. We illustrate relative observation utilization (pOBS) for nonspecific chest pain stratified by several hospital characteristics: teaching hospital status (defined by membership in the American Association of Medical Colleges' Council of Teaching Hospitals or home institutions to Accreditation Council for Graduate Medical Education accredited resi- dency programs) and hospital size (defined by bed count as small, medium or large per the Healthcare Cost Utilization Project's definition for the National Inpatient Sample) [11].

For nonspecific chest pain, we calculated the difference between average charges for an inpatient hospitalization and an observation visit by CCS conditions. We then modeled potential savings in charges and as a percent of charges if all hospitals had a minimum pOBS equal to the current median hospital or the current 75 th percentile hospital.

All $p$-values were two-tailed, and the $\alpha$-level was 0.05 . Data analysis was performed with SAS 9.1 (SAS, Cary, NC). The study was considered exempt from review by the hospital IRB.

\section{Results}

From 2003 to 2006 observation visits in Massachusetts increased by $3.9 \%$ [95\% confidence interval (CI): $3.8 \%$ to $4.0 \%$ ] from 128,825 to 133,859 , while inpatient hospitalizations increased by $1.3 \%$ (95\% CI $1.26 \%$ to $1.31 \%$ ) from 832,415 to 843,617 , and ED visits increased by $4.9 \%$ (95\% CI $4.95 \%$ to $5.00 \%$ ) from 2.78 million to 2.92 million. Nonspecific chest pain was the most frequently observed condition, with 85,843 evaluations accounting for $16.3 \%$ of statewide observation visits over the 4 -year period. The next most frequently observed conditions were: syncope (14,882 visits; $2.8 \%$ of all observation visits), abdominal pain $(13,573 ; 2.5 \%)$, fluid and electrolyte disorders $(12,121 ; 2.3 \%)$ and asthma $(8,491 ; 1.6 \%)$. There were 2,098 observation visits for congestive heart failure $(0.4 \%$ of total). Table 1 reports hospital utilization data for each clinical condition. 
Across Massachusetts, hospitals' pOBS rates varied by clinical condition (Table 1). Chest pain had the widest variation, from $25 \%$ to $95 \%$, while congestive heart failure had the least variation, $5 \%$ to $35 \%$. Figure 1 illustrates the variation in pOBS for nonspecific chest pain across Massachusetts hospitals regardless of teaching status or size.

From 2003 to 2006 , there was a $43.5 \%(95 \%$ CI $43.1 \%$ to $43.8 \%$ ) increase in observation visits for nonspecific chest pain, while inpatient hospitalizations declined by $6.3 \%$ (95\% CI $6.1 \%$ to $6.5 \%$ ). From 2003 to 2006, the relative frequency of observation diagnoses (pOBS) increased for nonspecific chest pain and syncope, while the relative frequency decreased for asthma and fluid and electrolyte disorders and did not change for CHF and abdominal pain (Table $1 ; P<0.001)$.
Observation charges for the median hospital are shown for each clinical condition in Table 1 . We modeled the impact of the variation in charges for nonspecific chest pain evaluation across Massachusetts hospitals in Table 2. The median hospital's charge for an observation visit in 2005 was $\$ 5,438$. Of the 74 hospitals reporting observation data for nonspecific chest pain in 2005, inpatient charge data were also available for 59 (median $\$ 7,011$ ) with a median charge $28 \%$ higher than for observation. The subset of hospitals analyzed in Table 2 had similar relative use of observation (pOBS $=57.5 \%$ versus $59.0 \%$ at all 74 hospitals). If the 29 hospitals with relative observation (pOBS) rates below the median hospital used observation at the same rate as the median and the remaining 30 hospitals were unchanged, then annual, statewide charges for nonspecific chest pain would decrease by $\$ 3.4$ million,
Fig. 1 Utilization of observation for nonspecific chest pain: Massachusetts 2003-2006

\section{a Stratified by Hospital Teaching Status}

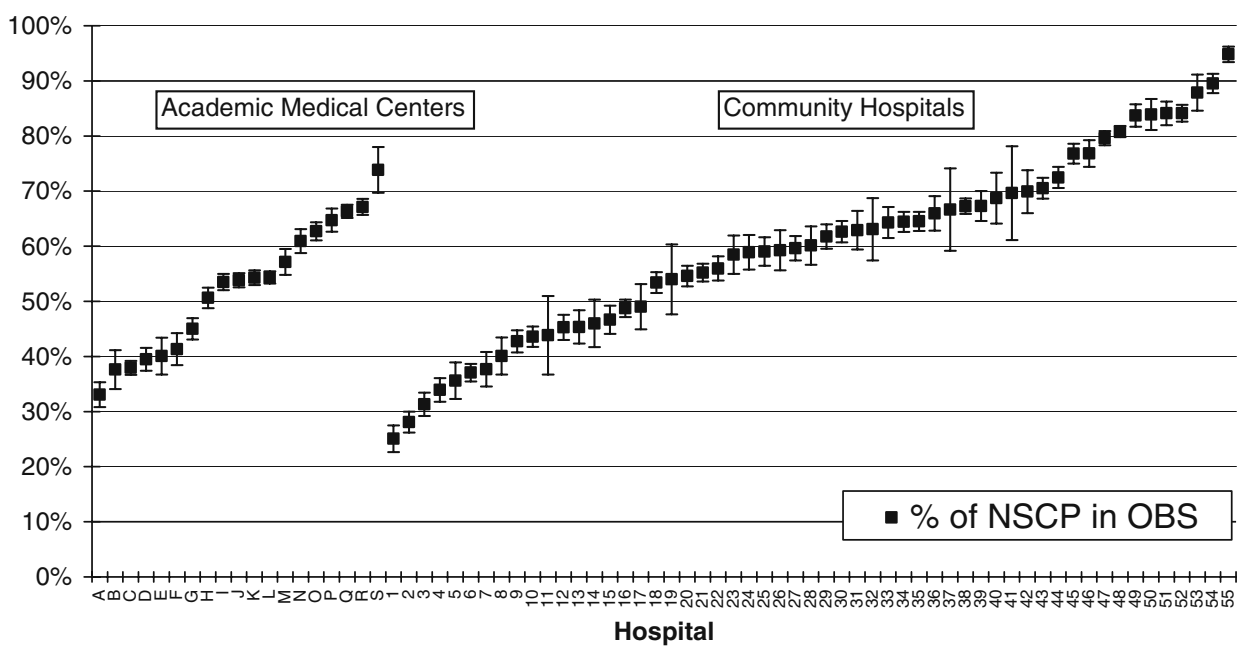

b Stratified by Hospital Size

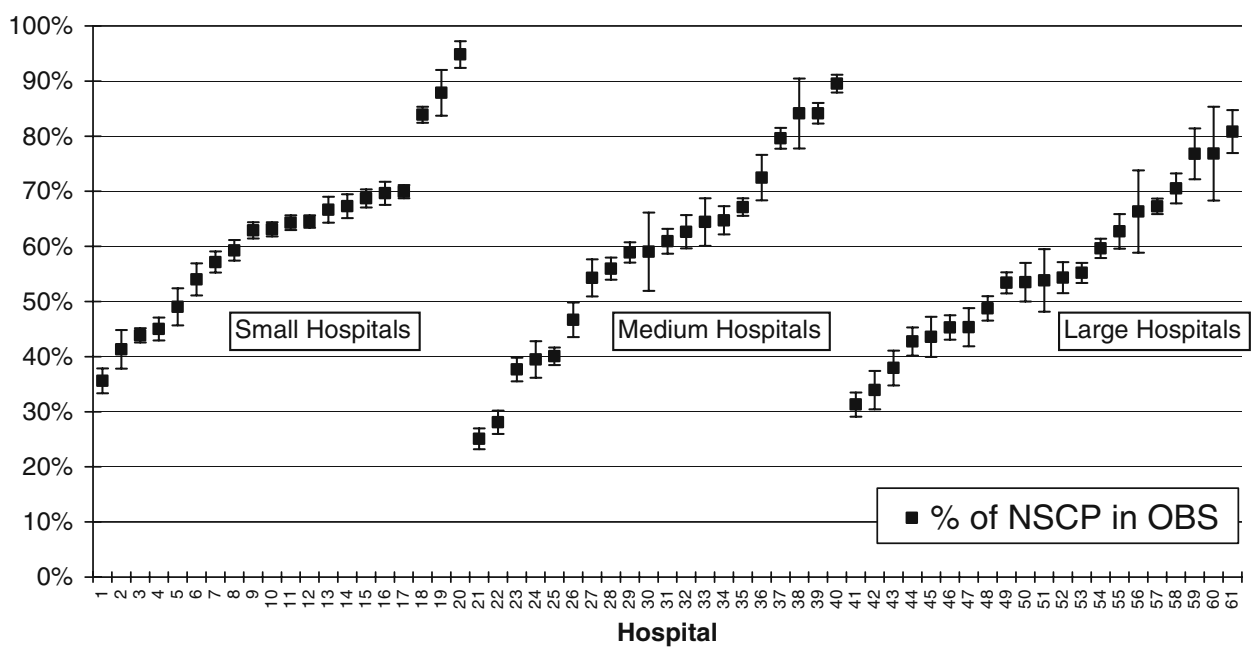

${ }^{*}$ NSCP $=$ Nonspecific chest pain, defined by clinical Classification System 102(6) 
Table 2 Effect of hospital variation on state-wide charges for nonspecific chest pain in 2005

*Charge projections done at hospital level assuming mean hospital charge for CCS 102

$* * 50$ th percentile $(\mathrm{pOBS}=58 \%)$ and 44 hospitals below the 75th percentile $(\mathrm{pOBS}=64 \%)$

\begin{tabular}{lcc}
\hline & Observation & Inpatient \\
\hline Total statewide evaluations at all 74 hospitals & 24,043 & 16,093 \\
Actual 2005 statewide charges & $\$ 151,026,323$ & Unknown \\
Total statewide evaluations at 59 hospitals reporting charge data & 19,596 & 14,895 \\
Actual 2005 charges at 59 hospitals reporting charge data & $\$ 122,800,528$ & $\$ 121,045,318$ \\
Scenario 1: All hospitals perform at least at 50th percentile (pOBS $\geq 58 \%)$ & \\
Projected evaluations & 21,737 & 12,754 \\
Projected charges & $\$ 136,751,072$ & $\$ 103,682,115$ \\
Potential statewide annual savings & $\$ 3,412,659$ & \\
Scenario 2: All hospitals perform at least at 75th percentile (pOBS $\geq 64 \%)$ & 11,611 \\
Projected evaluations & 22,880 & $\$ 94,458,641$ \\
Projected charges & $\$ 143,862,471$ & \\
Potential statewide annual savings & $\$ 5,524,734$ & \\
\hline
\end{tabular}

$1.4 \%$ of reported nonspecific chest pain charges. Similarly, if the 44 hospitals performing below the 75th percentile (pOBS $=63.4 \%$ ) performed at the 75 th percentile, then statewide charges for nonspecific chest pain would decrease by $\$ 5.5$ million (2.3\% of reported nonspecific chest pain charges).

\section{Discussion}

Hospital and ED utilization have received intense scrutiny as a significant driver of overall health care costs, yet use of observation services has been ignored [12]. We analyzed statewide, summary-level administrative data to document variation in the use of observation visits and observation charges relative to inpatient hospitalizations across six common emergency medical conditions. We found that the use of observation services grew at 3.9\%, more than inpatient hospitalization and less than ED visitation. These changes occurred while the population of Massachusetts was stable at 6.44 million over the study period [13]. Specifically, the utilization of observation services for the evaluation of nonspecific chest pain grew over $40 \%$ from 2003 to 2006, while the relative utilization of observation varied widely across hospitals, ranging from $25 \%$ to $95 \%$ of total inpatient and observation visits. Although we were unable to account for patient-level characteristics, wide variation persisted after stratification of hospitals by teaching status and hospital size.

The impact of observation use on statewide health care costs is difficult to estimate from publicly available administrative data. We demonstrated two scenarios, which estimate several million dollars in statewide savings to payers for chest pain, but this represents less than five percent of annual charges for chest pain. These estimates are based on hospital charges, which were $28 \%$ less for observation visits than for inpatient hospitalization. Our estimate is conservative, as hospitals disproportionately account overhead to outpatient units, so observation charges likely overstate costs, and accurate cost-to-charge ratios for observation are not available. Previous studies have documented larger differences in actual health care costs between inpatient and observation visits for chest pain, which would result in larger calculated savings [1, 2]. A more detailed model that quantifies the effects of increased observation utilization on multiple conditions based on actual cost data might yield a more compelling economic case for the efficiency of observation evaluation.

These pilot data provide an initial view of hospital observation use across one state. It is not clear whether the variation observed represents underuse, overuse or misuse. Future research should evaluate observation care in the context of inpatient hospitalization, emergency care and longer term patient outcomes. For example, with nonspecific chest pain, in addition to looking at observation use, one would need to look at ED discharge practices and proximate outcomes (e.g., 30-day missed acute myocardial infarction rate) to determine if the care pattern is safe and efficient. Although not directly studied, it is reasonable that an observation stay would be as safe as or safer than a similar inpatient admission as the duration in hospital is shorter and the quality of care has been shown to be equal or superior $[1,2]$. If observation care is not associated with worse in-hospital or proximate outpatient outcomes, then it may advance the value of health care delivery.

This pilot study has several limitations. We analyzed summary level administrative data, which precluded risk adjustment for patients' clinical or sociodemographic characteristics. However, the wide variation in observation use is difficult to explain by a patient's clinical condition alone, as illustrated by the presence of wide variation between similar hospitals types. Second, administrative data are created for billing rather than clinical or research purposes. Discharge codes are subject to biased coding patterns at the hospital or group level. For example, one ED 
or coding group may routinely diagnose patients with nonspecific chest pain as gastroesophageal reflux while another ED diagnoses chest pain. In the future, patient-level analysis and diagnosis-based sensitivity analyses should be performed to explore the effect of coding patterns on these findings. Third, we did not have a reliable method to determine if a hospital had a dedicated ED observation unit or clinical pathways for observation on inpatient wards; these are likely associated with observation use. Finally, these data are from a single state, limiting their generalizability. Massachusetts is a good state for pilot observation research as it was one of the first states to collect observation data and is one of less than ten states currently collecting such data.

Due to large and increasing costs associated with inpatient hospitalization, CMS and private payers have adopted hospital utilization measures as markers of efficiency, specifically focusing on rehospitalization rates and emergency department recidivism [12]. As future efforts are directed at reducing variation in health care utilization in the name of efficiency, acute care hospitalizations will be a primary focus, and the ED will increasingly be recognized as a locus of control. The wide variation in observation use relative to inpatient admission for emergency medical conditions in Massachusetts is intriguing and suggests the potential for efficiencies in health care delivery that deserve further investigation.

Funding/Conflict of Interest JDS serves on the Primary Care and Emergency Medicine Scientific Advisory Board for United Healthcare. SJB and AV have no conflict of interest or disclosures.

\section{References}

1. Graff LG (2009) Observation medicine: The healthcare system's tincture of time. American College of Emergency Physicians online publication. 176. Available at: http://www.acep.org/
WorkArea/DownloadAsset.aspx $\mathrm{id}=45885$. Accessed November 11,2009

2. Farkouh ME, Smars PA, Reeder GS et al (1998) A clinical trial of a chest-pain observation unit for patients with unstable angina. Chest pain evaluation in the emergency room (CHEER) investigators. N Engl J Med 339:1882-1888

3. Kohn LT, Corrigan JM, Donaldson MS (Institute of Medicine) (2000). To err is human: building a safer health system. National Academy Press, Washington, DC

4. Brennan TA, Leape LL, Laird NM et al (1991) Incidence of adverse events and negligence in hospitalized patients. Results of the Harvard Medical Practice Study I. N Engl J Med 324:370-376

5. Fisher ES, Wennberg JE, Stukel TA et al (2000) Associations among hospital capacity, utilization, and mortality of US Medicare beneficiaries, controlling for sociodemographic factors. Health Serv Res 34:1351-1362

6. Pitts SR, Niska RW, Xu J et al (2008) National hospital ambulatory medical care survey: 2006 emergency department summary. Natl Health Stat Report (7):1-38

7. Andrews RM, Elixhauser A (2007) The national hospital bill: Growth trends and 2005 update on the most expensive conditions by payer. December 2007. Available at AHRQ Website: http:// www.acep.org/WorkArea/DownloadAsset.aspx ?id=45885. Accessed November 11, 2009

8. Elixhauser A, Steiner C, Palmer L, Clinical classification software (CCS) (2008) Available at AHRQ website: http://www.acep.org/ WorkArea/DownloadAsset.aspx $\mathrm{id}=45885$. Accessed November 11,2009

9. Landon BE, Normand ST (2006) National voluntary consensus standards for ambulatory care: Measurement challenges in small group settings". Available at National Quality Forum website: http://www.acep.org/WorkArea/DownloadAsset.aspx?id=45885. Accessed November 11, 2009

10. Center for Medicare and Medicaid Services (2009) Acute care hospital inpatient prospective payment system: Fact sheet. Available at CMS website: http://www.acep.org/WorkArea/ DownloadAsset.aspx?id=45885. Accessed November 11, 2009

11. Healthcare Cost and Utilization Project (2008) NIS description of data elements: HOSP BEDSIZE. Available at HCUP website: http://www.acep.org/WorkArea/DownloadAsset.aspx?id=45885. Accessed November 11, 2009

12. National Priorities Partnership (2008) National priorities and goals: aligning our efforts to transform America's healthcare (2008). National Quality Forum, Washington

13. US Census Population Estimates (2010). Population change: April 1, 2000 to July 1, 2009. Available at: http://www.census.gov/ popest/states/files/NST-EST2009-popchg2000-2009.csv. Accessed April 3, 2010 